\title{
Strain-Induced Indirect to Direct Bandgap Transition in Multilayer $\mathrm{WSe}_{2}$
}

\author{
Sujay B. Desai, ${ }^{\dagger, \ddagger}$ Gyungseon Seol, ${ }^{\S}$ Jeong Seuk Kang, ${ }^{\dagger, \ddagger}$ Hui Fang, ${ }^{\dagger, \ddagger}$ Corsin Battaglia, ${ }^{\dagger, \ddagger}$ \\ Rehan Kapadia, ${ }^{\dagger, \ddagger}$ Joel W. Ager, Jing Guo, ${ }^{\S}$ and Ali Javey, ${ }^{\dagger},+$ \\ ${ }^{\dagger}$ Electrical Engineering and Computer Sciences, University of California, Berkeley, California 94720, United States \\ ${ }^{\ddagger}$ Materials Sciences Division, Lawrence Berkeley National Laboratory, Berkeley, California 94720, United States \\ ${ }^{\S}$ Department of ECE, University of Florida, Gainesville, Florida 32611-6130, United States
}

\section{Supporting Information}

\begin{abstract}
Transition metal dichalcogenides, such as $\mathrm{MoS}_{2}$ and $\mathrm{WSe}_{2}$, have recently gained tremendous interest for electronic and optoelectronic applications. $\mathrm{MoS}_{2}$ and $\mathrm{WSe}_{2}$ monolayers are direct bandgap and show bright photoluminescence (PL), whereas multilayers exhibit much weaker PL due to their indirect optical bandgap. This presents an obstacle for a number of device applications involving light harvesting or detection where thicker films with direct optical bandgap are desired. Here, we experimentally demonstrate a drastic enhancement in PL intensity for multilayer $\mathrm{WSe}_{2}$ (2-4 layers) under uniaxial tensile strain of up to $2 \%$. Specifically, the PL intensity of bilayer $\mathrm{WSe}_{2}$ is amplified by $\sim 35 \times$, making it comparable to that of an unstrained $\mathrm{WSe}_{2}$ monolayer. This drastic PL enhancement is attributed to an indirect to direct bandgap transition for strained bilayer $\mathrm{WSe}_{2}$, as confirmed by

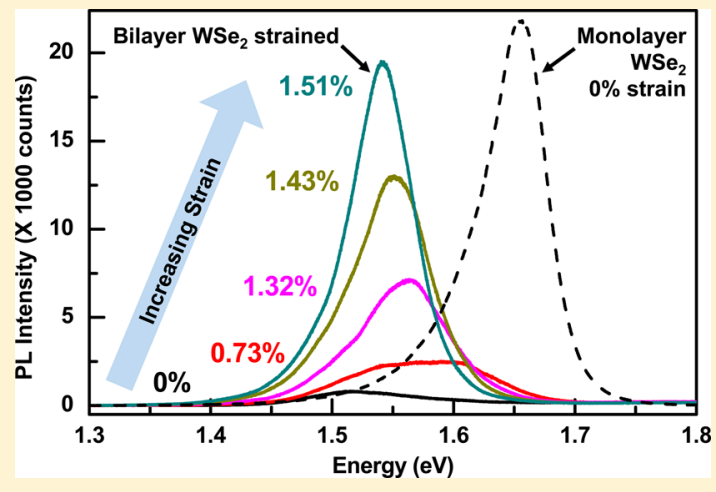
density functional theory (DFT) calculations. Notably, in contrast to $\mathrm{MoS}_{2}$ multilayers, the energy difference between the direct and indirect bandgaps of $\mathrm{WSe}_{2}$ multilayers is small, thus allowing for bandgap crossover at experimentally feasible strain values. Our results present an important advance toward controlling the band structure and optoelectronic properties of few-layer $\mathrm{WSe}_{2}$ via strain engineering, with important implications for practical device applications.
\end{abstract}

KEYWORDS: layered materials, uniaxial tensile strain, indirect to direct bandgap transition, photoluminescence, strain engineering

$\mathrm{T}$ ransition metal dichalcogenides (TMDCs) are a class of materials consisting of transition metals $\mathrm{M}-(\mathrm{Mo}, \mathrm{W}, \mathrm{Sn}$, etc.) covalently bonded to chalcogens $\mathrm{X}-(\mathrm{S}, \mathrm{Se}, \mathrm{Te})$ which arrange in stacked layers held together by van der Waals forces. Due to their layered structure and sizable bandgaps, semiconducting TMDCs such as $\mathrm{MoS}_{2}$ and $\mathrm{WSe}_{2}$ have established themselves as strong candidates for future electronic and optoelectronic applications. ${ }^{1-8}$ It has been shown that monolayer $\mathrm{MoS}_{2}$ and $\mathrm{WSe}_{2}$ exhibit a direct bandgap (K-K) with strong photoluminescence (PL). ${ }^{9-11}$ For example, monolayer $\mathrm{MoS}_{2}$ has a high PL quantum yield on the order of $\sim 4 \times 10^{-3}$ (ref 9), and monolayer $\mathrm{WSe}_{2}$ is found to exhibit greater PL intensity than $\mathrm{MoS}_{2}$ (ref 11). However, multilayers of these TMDCs have an indirect bandgap and show significantly weaker PL., ${ }^{9,12}$ The indirect nature of multilayer TDMCs therefore limits their application in optoelectronic devices such as light-emitting diodes, photodetectors, and lasers.

Strain can be used to modulate the band structure and engineer the properties of a material. Specifically, the lattice constant and van der Waals gap for TMDCs change by strain. This leads to a direct change in the electronic band structure and hence the energies of the conduction band $(\mathrm{CB})$ minima and valence band $(\mathrm{VB})$ maxima for the material. If the energy difference of the indirect and direct bandgaps is small, then it may be possible to achieve a crossover from one to the other using strain. For example, Ge shows an indirect to direct bandgap transition when strained due to the small difference of its two energy bandgaps. ${ }^{13,14}$ The effect of strain on TMDCs has been studied for $\mathrm{MoS}_{2}$ as a model TMDC material. ${ }^{15}$ However, for $\mathrm{MoS}_{2}$ multilayers, the direct and indirect bandgap differ by a large value (i.e., $\sim 300 \mathrm{meV}$ for bilayer $\left.\mathrm{MoS}_{2}\right)^{9}$ and hence no transition is seen on the application of up to $2.2 \%$ uniaxial tensile strain. ${ }^{15}$ In contrast to $\mathrm{MoS}_{2}, \mathrm{WSe}_{2}$ multilayers have a much smaller difference between the direct and indirect bandgaps, on the order of $40 \mathrm{meV}$ for bilayer $\mathrm{WSe}_{2}{ }^{16}{ }^{16}$ Thus, a crossover from indirect to direct bandgap should be possible in multilayer $\mathrm{WSe}_{2}$ for practically achievable strain values, similar to the case of Ge. However, to date, strain engineering of $\mathrm{WSe}_{2}$

Received: May 2, 2014

Revised: June 24, 2014

Published: July 2, 2014 

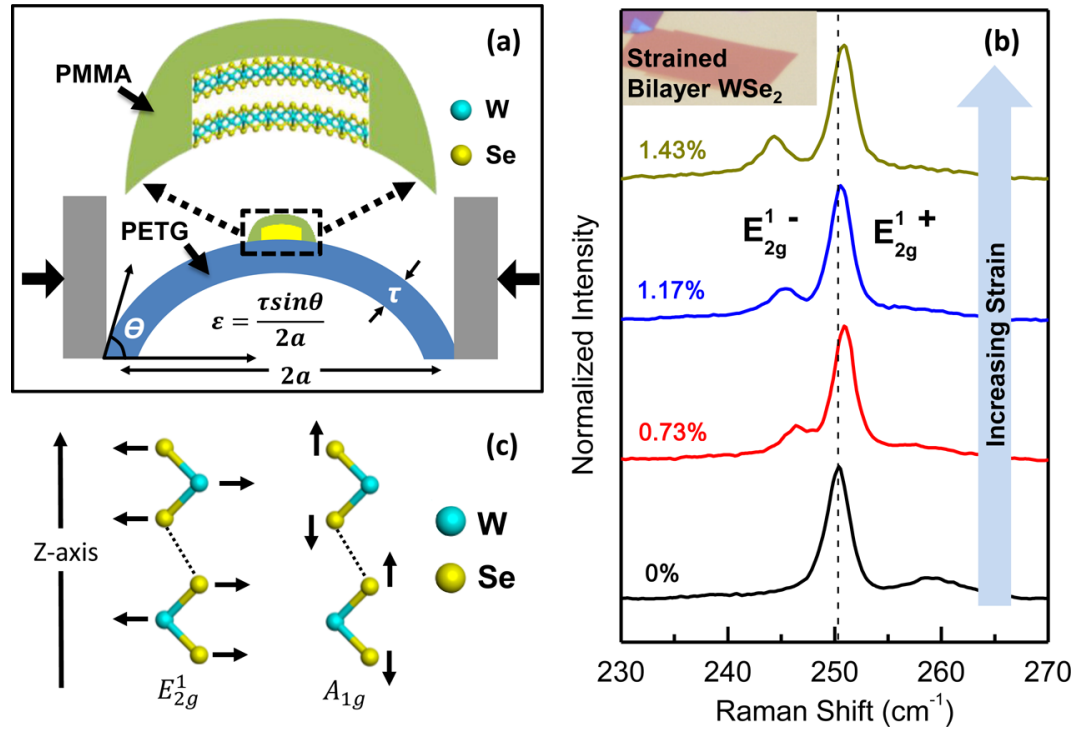

Figure 1. (a) Schematic of the two-point bending apparatus along with the method to compute strain. WSe $e_{2}$ flake encapsulated by PMMA is transferred onto flexible PETG. $\tau$ is the thickness of the PETG, $\varepsilon$ is the strain, $\theta$ is the angle of the tangent at the minimum strain point, and $2 a$ is the separation of the bent PETG. (b) Raman spectra for bilayer $W \mathrm{We}_{2}$ at different strain. $\mathrm{E}_{2 \mathrm{~g}}^{1}$ degeneracy breaks under strain and the mode splits into $\mathrm{E}_{2 \mathrm{~g}}^{1-}$ and $\mathrm{E}_{2 g}^{1+}$ which show a left and right shift from the original Raman peak. Inset shows the optical microscope image of the bilayer WSe $e_{2}$ flake used in this study. (c) The schematic of $\mathrm{E}_{2 \mathrm{~g}}^{1}$ and $\mathrm{A}_{1 \mathrm{~g}}$ Raman active modes of $\mathrm{WSe}_{2}$.
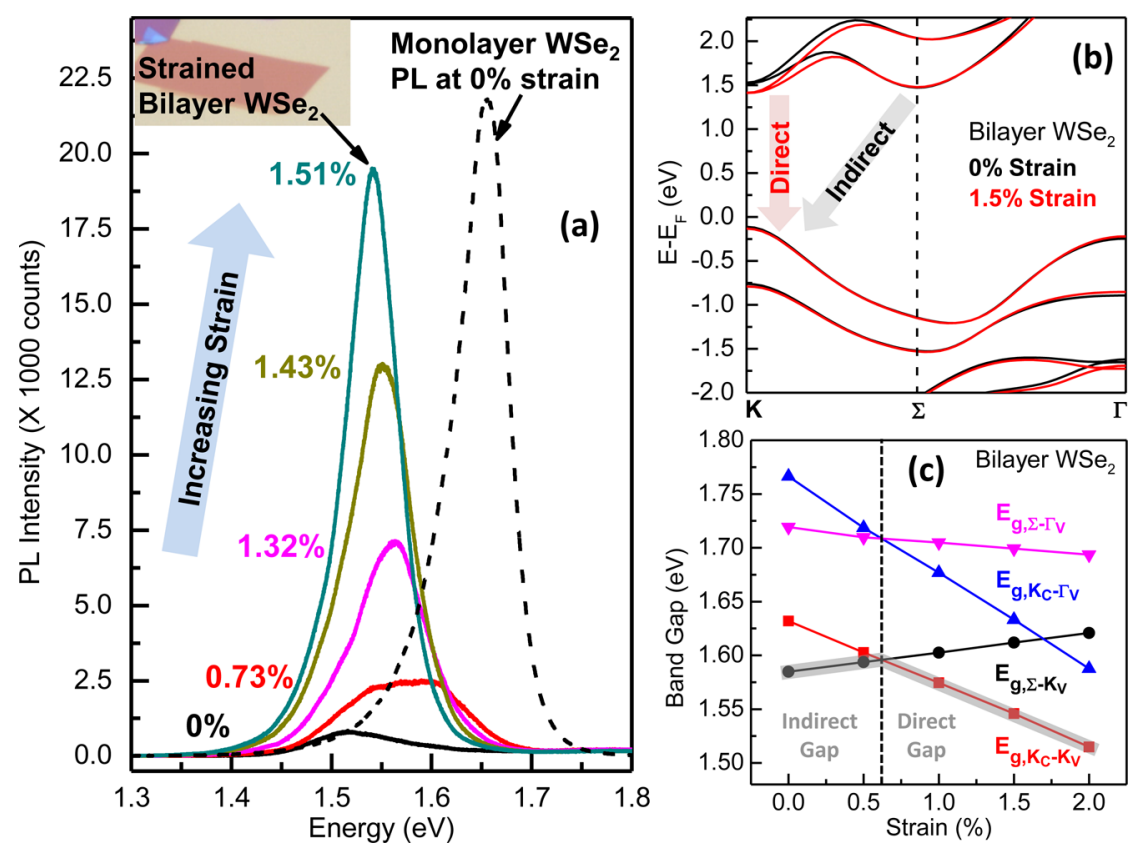

Figure 2. (a) Bilayer $\mathrm{WSe}_{2} \mathrm{PL}$ spectra at different strain. PL of unstrained monolayer $\mathrm{WSe}_{2}$ on $\mathrm{SiO}_{2}$ is shown for comparison. Inset shows the optical microscope image of the bilayer WSe $e_{2}$ flake used in this study. (b) Electronic band structure for bilayer WSe $\mathrm{e}_{2}$ with and without strain using HSEDFT. CB and VB changes under strain are clearly seen with the K point CB minima decreasing drastically in energy whereas the $\Sigma$ point CB minima increases slightly, thus showing the indirect to direct bandgap transition. (c) Bandgap energies for different transitions of bilayer WSe ${ }_{2}$ calculated using HSE-DFT.

multilayers has not been explored, and this unique property of $\mathrm{WSe}_{2}$ has not been exploited.

In this work, we experimentally demonstrate a drastic increase in PL intensity for multilayer $\mathrm{WSe}_{2}$ (2-4 layers thick) by applying uniaxial tensile strain of up to $\sim 2 \%$. The maximum PL amplification observed at this strain for bilayer $\mathrm{WSe}_{2}$ is $\sim 35 \times$. The PL intensity of strained bilayer $\mathrm{WSe}_{2}$ is comparable to that of an unstrained $\mathrm{WSe}_{2}$ monolayer measured under similar experimental conditions. A significant $\mathrm{PL}$ intensity increase is also detected for trilayer and quadlayer $\mathrm{WSe}_{2}$ upon uniaxial strain. Density functional theory (DFT) calculations further confirm a strain-induced indirect to direct transition in multilayer $\mathrm{WSe}_{2}$ due to opposite shifts of the $\mathrm{CB}$ minima at the $\mathrm{K}$ and $\Sigma$ points in the reciprocal space, explaining the PL emission enhancement observed in the experiment.

A two-point bending method is used to apply uniaxial tensile strain to the $\mathrm{WSe}_{2}$ flakes, as illustrated in Figure 1a. $\mathrm{WSe}_{2}$ is first exfoliated onto a $\mathrm{Si} / \mathrm{SiO}_{2}(260 \mathrm{~nm}$ thick) substrate, which is 
mapped using an optical microscope $\mathrm{e}^{17,18}$ (and atomic force microscope) to find flakes with the desired number of layers (1-4). Each flake is then transferred to a clear and flexible polyethylene terephthalate glycol-modified (PETG) substrate of $1.5 \mathrm{~mm}$ thickness using poly(methyl methacrylate) (PMMA) as the transfer medium. ${ }^{19}$ The PMMA caps the flake on the PETG and acts as a clamp, thus enabling the application of large strain. The bent PETG is approximated by a circular arc for calculation of strain which can be computed as shown in Figure 1a (more details on the sample preparation and strain calculation can be found in the Supporting Information). In addition to the $\mathrm{WSe}_{2}$ flake of interest, a $\mathrm{MoS}_{2}$ monolayer is also transferred onto the same PETG in close proximity to the $\mathrm{WSe}_{2}$ flake. This $\mathrm{MoS}_{2}$ monolayer serves as an internal reference for predicting strain using data in ref 15 , in addition to calculating strain from the bending geometry. Supporting Information Figure S1a and $\mathrm{b}$ show the evolution of the PL and Raman data for the monolayer $\mathrm{MoS}_{2}$ reference with strain. The strain predicted using this reference sample shows an almost 1:1 correlation with the calculated strain from the bending geometry as is shown in Supporting Information Figure S1c. Raman and PL measurements (Horiba Scientific LabRAM HR 800) were taken for strained $\mathrm{WSe}_{2}$ at several representative spots with site-to-site variation arising from local strain profile of the flakes (see Supporting Information Figure S2). A $532 \mathrm{~nm}$ laser with $8-80 \mu \mathrm{W}$ power and a spot size of $\sim 0.5 \mu \mathrm{m}$ was used. The lowest laser power giving appreciable signal-to-noise ratio (SNR) is used in order to prevent damage to the TMDC flakes while making sure that the Raman and PL peaks do not shift due to heating effects in the sample.

Figure $1 \mathrm{~b}$ shows the Raman spectra for bilayer $\mathrm{WSe}_{2}$ under different uniaxial tensile strain up to $\sim 1.43 \%$. The atomic vibrations corresponding to the $E_{2 g}^{1}$ (in-plane) and $A_{1 g}$ (out-ofplane) Raman modes for $\mathrm{WSe}_{2}$ are shown in Figure $1 \mathrm{c}$. $^{16,20}$ Under application of strain, the degeneracy of the $\mathrm{E}_{2 \mathrm{~g}}^{1}$ mode is broken and the peak splits into two peaks, $\mathrm{E}_{2 \mathrm{~g}}^{1+}$ and $\mathrm{E}_{2 \mathrm{~g}}^{1-}$. The two peaks move in opposite directions from the original peak, with $E_{2 g}^{1-}$ left shifting by a large amount compared to the $E_{2 g}^{1+}$ peak, which shows a right shift. Raman shift is an indicator of the amount by which the lattice constant is changed. Also a left shift indicates tensile strain or increase in the lattice constant whereas a right shift indicates compressive strain or a reduction in the lattice constant. Thus, $\mathrm{E}_{2 \mathrm{~g}}^{1-}$ and $\mathrm{E}_{2 \mathrm{~g}}^{1+}$ correspond to the vibrational modes parallel and perpendicular to the direction of strain. The peak splitting increases as strain is increased. Thus, Raman splitting and shift are clear indicators of strain application on the sample.

Figure 2a depicts the evolution of the bilayer $\mathrm{WSe}_{2} \mathrm{PL}$ with strain. A drastic enhancement in PL intensity for bilayer $\mathrm{WSe}_{2}$ is clearly seen as the uniaxial tensile strain is increased. The peak at no strain is broad and the PL peak intensity is more than an order of magnitude less than that for unstrained monolayer $\mathrm{WSe}_{2}$, which is also shown in Figure $2 \mathrm{a}$ for reference. As strain increases, a second peak at higher energy corresponding to the direct valley transition $\left(K_{\mathrm{C}}-K_{\mathrm{V}}\right)$ appears. (In the notation, $K_{\mathrm{C}}, \Sigma_{\mathrm{V}}$, etc., the subscript $\mathrm{C}$ and $\mathrm{V}$ represent the $\mathrm{CB}$ minima and $\mathrm{VB}$ maxima of the electronic band structure, respectively, with the corresponding position in the reciprocal space given by $\mathrm{K}, \Sigma, \Gamma$, etc. Refer to Supporting Information Figure S3 for more information.) This peak shows a red shift with increasing strain and at the same time the PL intensity increases significantly. Simultaneously, we also observe the peak corresponding to the indirect valley transition $\left(\Sigma_{\mathrm{C}}-K_{\mathrm{V}}\right)$ showing a small blue shift. At $\sim 1.5 \%$ strain, the bilayer PL intensity is amplified by $\sim 25 \times$. The full width half-maximum (FWHM) also decreases from $110 \mathrm{meV}$ at $0 \%$ strain to $62 \mathrm{meV}$ at $1.5 \%$ strain which is comparable to the FWHM for unstrained monolayer $\mathrm{WSe}_{2}(61.6 \mathrm{meV})$. The $\mathrm{PL}$ signal from the strained bilayer $\mathrm{WSe}_{2}$ is comparable to that from the unstrained monolayer $\mathrm{WSe}_{2}$ measured under identical laser power and acquisition time conditions.

The drastic increase in the PL intensity and reduction of FWHM for bilayer $\mathrm{WSe}_{2}$ under strain can be understood with the help of DFT calculations. In this study DFT calculations were performed using the projector-augmented wave (PAW) pseudopotential through the Vienna ab initio simulation package (VASP). ${ }^{21,22}$ For the atomic structural relaxation, generalized gradient approximation (GGA) method was used with Perdew-Burke-Ernzerhof (PBE) exchange-correlation functional. ${ }^{23}$ The Brillouin-zone sampling was done by the $9 \times$ $9 \times 1$ Monkhorst-Pack scheme. It is well-known that the DFT calculation within GGA underestimates the bandgap of a semiconductor, thus, to obtain more accurate $\mathrm{E}-k$ relation, Heyd-Scuseria-Ernzerhof (HSE) exchange correlation potential with spin orbit coupling was used to model the band structure with increased $k$-point sampling of $15 \times 15 \times 1$ (ref 24). HSE gives a larger bandgap than LDA and GGA simulations, but a smaller bandgap than GW calculations. It has also been indicated that the optical gap is related to the GW bandgap subtracting the exciton binding energy. To verify, GW quasiparticle (QP) calculation, non-self-consistent $\mathrm{G}_{0} \mathrm{~W}_{0}$ was also performed (details can be found in the Supporting Information Figure S7). ${ }^{25}$ The band-structure by the $\mathrm{G}_{0} \mathrm{~W}_{0}$ calculations has an approximately $k$-independent shift of the conduction bands with regard to the valence bands $(0.65 \mathrm{eV}$ increase between $K_{\mathrm{C}}-K_{\mathrm{V}}$ ), without changing the direct or indirect bandgap feature (see Supporting Information Figure S7), similar to what has been shown before with or without strain. $^{26}$ The GW bandgap is larger than HSE bandgaps. An exciton binding energy of about $1 \mathrm{eV}$ has been reported for monolayer dichalcogenide materials, but with uncertainty. The value varies by about a factor of 2 if different $k$ point grids are used in simulation, ${ }^{26}$ and it decreases by about a factor of 2 from monolayer to bilayer. ${ }^{27}$ It is also dependent on the surrounding electrostatic environment. The uncertainty of the exciton binding energy makes a comparison to experiment difficult. On the other hand, it is found that the PL peak energy agrees with the bandgap of the HSE calculation. This is a coincidence, which could be due to cancellation of the blue shift by GW correction and red shift by exciton binding. Nevertheless, instead of introducing fitting parameters for uncertainty of the exciton binding energy, the band-structures from the HSE calculations with spin orbit coupling (SOC) are presented next because we are mainly interested in the indirect to direct bandgap transitions with strain applied. Structure relaxation was first performed to determine the relaxed atomistic structure in the presence of uniaxial strain, and the band structure in the presence of strain was subsequently calculated.

Figure $2 \mathrm{~b}$ compares the electronic band structure for bilayer $\mathrm{WSe}_{2}$ at $0 \%$ and $1.5 \%$ uniaxial tensile strain calculated within DFT using the HSE exchange-correlation functional. Inspection of Figure $2 \mathrm{~b}$ reveals that $1.5 \%$ strain lowers the energy of the $\mathrm{CB}$ minima at the (6-fold degenerate) $\mathrm{K}$ points by $86 \mathrm{meV}$ resulting in a transition from indirect to direct bandgap. The energy of the CB minima at the $\Sigma$ points interestingly shows an 

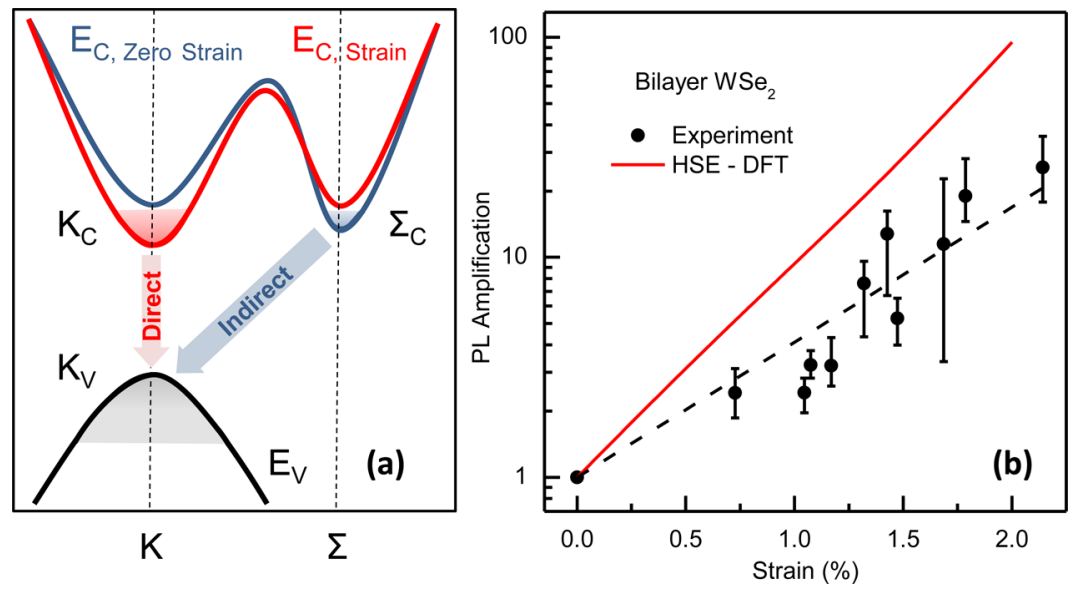

Figure 3. (a) Schematic band structure, qualitatively showing occupancy of $K_{\mathrm{C}}$ and $\Sigma_{\mathrm{C}} \mathrm{CB}$ minima at strain and zero-strain conditions and under illumination. (b) Bilayer WSe $\mathrm{WL}_{2}$ amplification versus strain. Experimental data for multiple WSe $\mathrm{e}_{2}$ samples measured at several spots on the flake show increasing PL amplification with increasing strain. Theory prediction using HSE-DFT is consistent with the experimental trend. Symbols represent average PL amplification, whereas the error bars represent the maximum and minimum error.

increase of $27 \mathrm{meV}$ at $1.5 \%$ strain. The transition from indirect to direct bandgap predicted by DFT thus qualitatively explains the $25 \times$ increase in PL intensity observed experimentally at $\sim 1.5 \%$ strain (Figure $2 \mathrm{a}$ ).

The $\mathrm{CB}$ minima at the $\mathrm{K}$ points of $\mathrm{WSe}_{2}$ exhibit dominant $\mathrm{W}$ $\mathrm{d}_{\mathrm{Z}^{2}}$ orbital character (refer to Supporting Information Figure S4). Under strain, the interlayer $\mathrm{W}-\mathrm{W}$ distance changes and so does the overlap of the orbitals. As a result, the energy of the $\mathrm{CB}$ minima at the K-points changes by a large amount leading to a crossover from indirect to direct bandgap. The different bandgap energies as calculated using DFT are compiled in Figure 2c. The transition from indirect to direct bandgap is predicted to happen at $\sim 0.625 \%$ strain. Experimentally, the transition is observed at a higher strain value, however, the trend predicted by DFT is consistent with the experimental data. This discrepancy between DFT and the experimental data can be attributed to the underestimation of bandgap from DFT calculations or from a difference between applied and actual strain due to local strain effects as discussed in Supporting Information Figure S2.

To understand the PL intensity amplification quantitatively, the electron occupancy at the direct $\mathrm{CB}$ valleys at the K-points must be considered. Figure $3 a$ schematically illustrates the occupancy of the CB and the VB under laser illumination. The Fermi level splits into quasi-Fermi levels for electrons, $F_{n}$ and holes, $\mathrm{F}_{\mathrm{p}}$ respectively. Tensile strain changes the $\mathrm{CB}$ minima, with the direct $\mathrm{CB}$ valleys $K_{\mathrm{C}}$ moving down while the indirect $\mathrm{CB}$ valleys $\Sigma_{\mathrm{C}}$ moving up in energy. The occupancy of electrons in each valley, therefore, correspondingly changes with strain.

We make a set of simplifying assumptions to arrive at the theoretical prediction. To first order, we assume there is no radiative recombination from the indirect transition $\left(\Sigma_{\mathrm{C}}-K_{\mathrm{V}}\right)$ and all the radiative recombination is from the direct transition $\left(K_{\mathrm{C}}-K_{\mathrm{V}}\right)$ only. This is a fair assumption given that a radiative recombination event for the indirect bandgap requires a phonon for momentum conservation, which makes the probability of the event very small compared to radiative recombination for the direct bandgap $\left(K_{\mathrm{C}}-K_{\mathrm{V}}\right)$. Next, the internal radiative efficiency $\eta_{\text {int }}$ for the $K_{\mathrm{C}}-K_{\mathrm{V}}$ transition is given by eq 1

$$
\eta_{\text {int }}=\frac{A n p}{A n p+B n p^{2}+C n}=\frac{A p}{A p+B p^{2}+C}
$$

Here, Anp represents the radiative recombination rate given by the product of the electron concentration $n$ in the $\mathrm{CB}$ valley, the majority hole concentration $p$ in the $\mathrm{VB}$, and the rate constant $A$. The other two terms, $B$ and $C$ represent the nonradiative recombination rate constants for Auger and Shockley-Read-Hall (SRH) processes, respectively. ${ }^{28}$ We see that $\eta_{\text {int }}$ depends only on the majority carrier concentration, and we assume the rate constants $A, B$, and $C$ to be constant with strain. The PL intensity is given by eq 2

$$
P L \propto\left[\eta_{i n t} \times \frac{n(D G)}{n(D G)+n(I G)} \times(n(D G)+n(I G))\right]
$$

where $n(D G)$ and $n(I G)$ are the number of electrons in the direct and indirect $\mathrm{CB}$ valley, respectively. Thus, the PL intensity is proportional to the fraction of radiative recombination from the direct valley, the fraction of carriers in the direct valley, and the total number of carriers generated by laser illumination. PL amplification (ratio of the PL peak intensity at nonzero strain to that at zero strain) can thus be described by eq 3

$$
\text { PL amplification }=\frac{n(D G)_{\text {strain }}}{n(D G)_{\text {zero strain }}}
$$

Using the Boltzmann approximation for calculating carrier concentrations we get

$$
\text { PL amplification }=\frac{e^{\left(\frac{-\mathrm{E}_{\mathrm{G}}(D G)_{\text {strain }}}{k T}\right)}}{e^{\left(\frac{-\mathrm{E}_{\mathrm{G}}(D G)_{\text {zerostrain }}}{k T}\right)}}
$$

Here, $\mathrm{E}_{\mathrm{G}}(D G)_{\text {zero strain }}$ and $\mathrm{E}_{\mathrm{G}}(D G)_{\text {strain }}$ are the direct bandgap $(D G)$ values at zero strain and nonzero strain conditions, respectively. These are taken from the DFT calculations for different strain points, and thus, we get the theoretical prediction of PL amplification. Because the CB valley shifts linearly with strain, the PL amplification changes exponentially with strain, as indicated by the red line in Figure $3 \mathrm{~b}$. Figure $3 \mathrm{~b}$ also shows the PL amplification vs strain for multiple bilayer $\mathrm{WSe}_{2}$ samples for different representative spots (represented by 
the error bars) along with the experimental data fit. The theoretical prediction using DFT is consistent with the experimental data, with only a slightly steeper slope. This difference between experiment and calculation can be due to the reasons highlighted earlier and also due to the simplifying assumptions related to the rate constants $A, B$, and $C$ in eq 1 .

We also examined the effect of strain on different thicknesses of $\mathrm{WSe}_{2}$. Figure $4 \mathrm{a}-\mathrm{b}$ show the PL and Raman data for
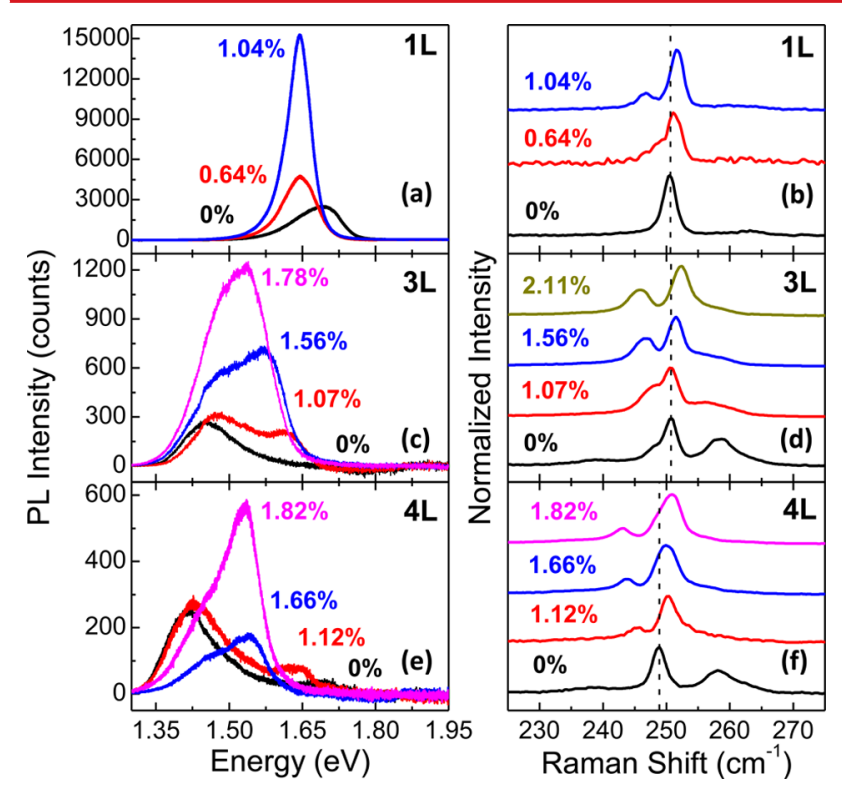

Figure 4. PL and Raman spectra at different strain for $(a-b)$ Monolayer, $(\mathrm{c}-\mathrm{d})$ Trilayer and $(\mathrm{e}-\mathrm{f})$ Quadlayer $\mathrm{WSe}_{2}$. In (c) and (e), a second peak corresponding to $K_{\mathrm{C}}-K_{\mathrm{V}}$ transition is seen at higher energies which red shifts with strain. $\Sigma_{\mathrm{C}}-K_{\mathrm{V}}$ peak blue shifts by a slight amount. Raman spectra in all cases show splitting under strain.

monolayer $\mathrm{WSe}_{2}$ under strain. Raman spectra clearly show the splitting and shift of the Raman modes as is observed for bilayer $\mathrm{WSe}_{2}$ indicating application of strain. PL for monolayer $\mathrm{WSe}_{2}$ shows a drastic increase with strain. This indicates that monolayer $\mathrm{WSe}_{2}$ remains direct bandgap even under uniaxial tensile strain and the difference between the indirect and direct $\mathrm{CB}$ minima increases further, as predicted by eq 4 . The results are consistent with the HSE-DFT analysis shown in Supporting Information Figure $\mathrm{S} 5 \mathrm{a}-\mathrm{b}$ and are in stark contrast to monolayer $\mathrm{MoS}_{2}$ behavior which in fact undergoes a direct to indirect transition on application of uniaxial tensile strain. ${ }^{15}$ The PL and corresponding Raman spectra at different strain for trilayer and quadlayer $\mathrm{WSe}_{2}$ are shown in Figure $4 c-d$ and Figure $4 \mathrm{e}-\mathrm{f}$, respectively. The background PL from the PMMA and PETG is subtracted from the trilayer and quadlayer $\mathrm{WSe}_{2}$ PL. The PL intensity gets amplified with strain for both trilayer and quadlayer. We also see a second peak corresponding to the direct gap appearing at higher energy which shows a red shift with increasing strain. Thus, all results and trends are consistent with earlier observations for bilayer $\mathrm{WSe}_{2}$. The decreasing PL amplification as a function of number of layers, for a given strain, is an indicator that the direct to indirect $C B$ valley difference increases with thickness. ${ }^{16}$

The PL trends of Figure 2 and Figure 4 together as a function of layer thickness can be understood by looking at the relative occupancies of the $K_{\mathrm{C}}((n(D G)) /(n(D G)+n(I G))$ and $\Sigma_{\mathrm{C}}((n(I G)) /(n(D G)+n(I G))$ valleys when illuminated by the laser as shown in Figure 5a. The peak values (for $\mathrm{WSe}_{2} 2-4$
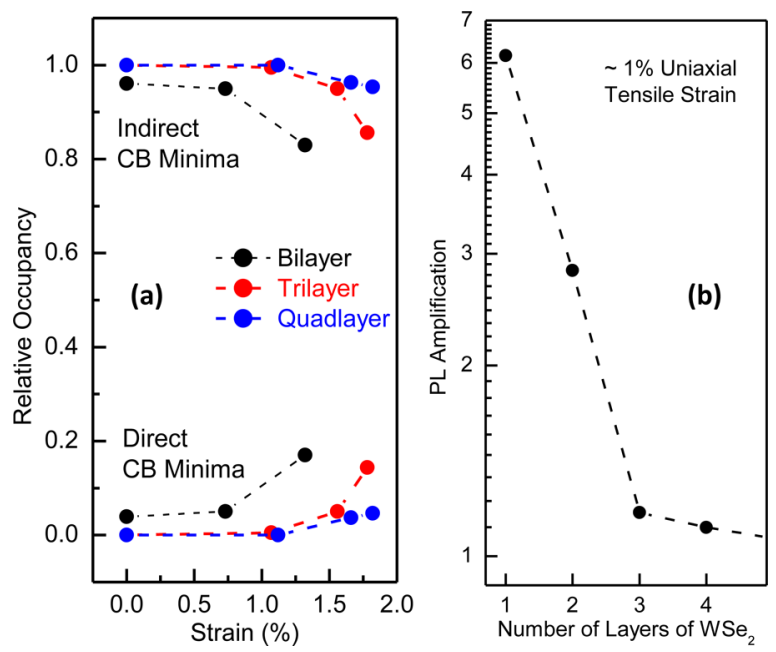

Figure 5. (a) Relative occupancy of the direct and indirect CB minima for bilayer, trilayer and quadlayer $\mathrm{WSe}_{2}$ as a function of strain. Peak values for occupancy calculations are extracted from the PL data. (b) PL amplification at $1 \%$ strain vs number of layers. PL amplification reduces with layer number since the direct and indirect bandgap difference at zero strain increases with layer number.

layers) are extracted from the PL data of Figure $2 \mathrm{a}$ and Figure $4 c$, e. As the $K_{C}-\Sigma_{C}$ difference reduces with increasing strain, the PL increases drastically resulting in the two peaks merging. From the occupancy plots, we can clearly understand the dependence of the critical strain required to bring about an indirect to direct bandgap crossover, on the number of layers. Bilayer $\mathrm{WSe}_{2}$ shows an increase in the $K_{\mathrm{C}}$ occupancy at a lower strain as compared to trilayer. The same trend is valid between trilayer and quadlayer $\mathrm{WSe}_{2}$. Figure $5 \mathrm{~b}$ shows the PL amplification at $\sim 1 \%$ strain as a function of layer number. The PL amplification achievable for a given strain value reduces as layer number increases. Supporting Information Figure S6a and $b$ show the PL and Raman spectra at different strain values for bulk WSe $e_{2}(\sim 30 \mathrm{~nm})$. We see a slight Raman peak splitting for bulk $\mathrm{WSe}_{2}$ but no significant change in the PL spectrum. This is consistent with the fact that the difference of $\sim 200 \mathrm{meV}$ between the indirect and the direct CB minima for bulk $\mathrm{WSe}_{2}$ at zero strain ${ }^{16}$ is so large that it is difficult to induce a crossover from indirect to direct bandgap with the maximum strain achievable by the experiment. We thus see a drastic increase in the PL intensity only for few-layer (1-4) $\mathrm{WSe}_{2}$ samples due to limitation of the maximum strain applicable by the experimental setup.

In conclusion, $\mathrm{WSe}_{2}$ multilayers are shown to exhibit drastic increase in PL intensity under the application of uniaxial tensile strain. Multilayer $\mathrm{WSe}_{2}$ with a direct bandgap is experimentally demonstrated and predicted by DFT by applying tensile strain. Moving forward, band structure and bandgap engineering with strain can be used as a method to change the mobility of carriers in $\mathrm{WSe}_{2}$. At the same time, increased PL emission properties make strained multilayer $\mathrm{WSe}_{2}$ a promising contender for optoelectronic devices. In addition to emission properties, future absorption studies under strain would provide great insight into the potential use of $\mathrm{WSe}_{2}$ in applications like photovoltaics and photodetectors. 


\section{ASSOCIATED CONTENT}

\section{S Supporting Information}

Sample preparation method. Strain calculations and $\mathrm{MoS}_{2}$ internal reference. Local strain effects and reversibility of strain. Density functional theory analysis of $\mathrm{WSe}_{2}$ under uniaxial tensile strain. Effect of uniaxial tensile strain on monolayer $\mathrm{WSe}_{2}$. Raman and PL spectra at different strain for bulk WSe $\mathrm{W}_{2}$. This material is available free of charge via the Internet at http://pubs.acs.org.

\section{AUTHOR INFORMATION}

\section{Corresponding Author}

*E-mail: ajavey@eecs.berkeley.edu.

\section{Notes}

The authors declare no competing financial interest.

\section{ACKNOWLEDGMENTS}

This work was supported by the Director, Office of Science, Office of Basic Energy Sciences, Material Sciences and Engineering Division of the U. S. Department of Energy under Contract No. DE-AC02-05CH11231. Strain measurement setup was built through funding by Intel. The authors wish to acknowledge Joint Center for Artificial Photosynthesis (JCAP), Lawrence Berkeley National Laboratory, for providing access to the Raman and PL measurement tools. The authors thank Dr. K. T. Lam of University of Florida for technical discussions.

\section{REFERENCES}

(1) Novoselov, K. S.; Jiang, D.; Schedin, F.; Booth, T. J.; Khotkevich, V. V.; Morozov, S. V.; Geim, A. K. Proc. Natl. Acad. Sci. U. S. A. 2005, 102 (30), 10451-3.

(2) Radisavljevic, B.; Radenovic, A.; Brivio, J.; Giacometti, V.; Kis, A. Nat. Nanotechnol. 2011, 6 (3), 147-50.

(3) Fang, H.; Chuang, S.; Chang, T. C.; Takei, K.; Takahashi, T.; Javey, A. Nano Lett. 2012, 12 (7), 3788-3792.

(4) Wang, Q. H.; Kalantar-Zadeh, K.; Kis, A.; Coleman, J. N.; Strano, M. S. Nat. Nano 2012, 7 (11), 699-712.

(5) Butler, S. Z.; Hollen, S. M.; Cao, L.; Cui, Y.; Gupta, J. A.; Gutiérrez, H. R.; Heinz, T. F.; Hong, S. S.; Huang, J.; Ismach, A. F.; Johnston-Halperin, E.; Kuno, M.; Plashnitsa, V. V.; Robinson, R. D.; Ruoff, R. S.; Salahuddin, S.; Shan, J.; Shi, L.; Spencer, M. G.; Terrones, M.; Windl, W.; Goldberger, J. E. ACS Nano 2013, 7 (4), 2898-2926.

(6) Chhowalla, M.; Shin, H. S.; Eda, G.; Li, L. J.; Loh, K. P.; Zhang, H. Nat. Chem. 2013, 5 (4), 263-75.

(7) Xu, M.; Liang, T.; Shi, M.; Chen, H. Chem. Rev. 2013, 113 (5), 3766-98.

(8) Jariwala, D.; Sangwan, V. K.; Lauhon, L. J.; Marks, T. J.; Hersam, M. C. ACS Nano 2014, 8 (2), 1102-1120.

(9) Mak, K. F.; Lee, C.; Hone, J.; Shan, J.; Heinz, T. F. Phys. Rev. Lett. 2010, 105, 136805.

(10) Splendiani, A.; Sun, L.; Zhang, Y.; Li, T.; Kim, J.; Chim, C.-Y.; Galli, G.; Wang, F. Nano Lett. 2010, 10 (4), 1271-1275.

(11) Tonndorf, P.; Schmidt, R.; Böttger, P.; Zhang, X.; Börner, J.; Liebig, A.; Albrecht, M.; Kloc, C.; Gordan, O.; Zahn, D. R. T.; Michaelis de Vasconcellos, S.; Bratschitsch, R. Opt. Expr. 2013, 21 (4), 4908-4916.

(12) Ellis, J. K.; Lucero, M. J.; Scuseria, G. E. Appl. Phys. Lett. 2011, 99 (26), 261908.

(13) Cheng, T.-H.; Peng, K.-L.; Ko, C.-Y.; Chen, C.-Y.; Lan, H.-S.; Wu, Y.-R.; Liu, C. W.; Tseng, H.-H. Appl. Phys. Lett. 2010, 96 (21), -

(14) Hoshina, Y.; Iwasaki, K.; Yamada, A.; Konagai, M. Jpn. J. of Appl. Phys. 2009, 48 (4S), 04C125.

(15) Conley, H. J.; Wang, B.; Ziegler, J. I.; Haglund, R. F.; Pantelides, S. T.; Bolotin, K. I. Nano Lett. 2013, 13 (8), 3626-3630.
(16) Sahin, H.; Tongay, S.; Horzum, S.; Fan, W.; Zhou, J.; Li, J.; Wu, J.; Peeters, F. M. Phys. Rev. B: Condens. Matter Mater. Phys. 2013, 87 (16), 165409.

(17) Castellanos-Gomez, A.; Agrait, N.; Rubio-Bollinger, G. Appl. Phys. Lett. 2010, 96 (21), 213116.

(18) Benameur, M. M.; Radisavljevic, B.; Héron, J. S.; Sahoo, S.; Berger, H.; Kis, A. Nanotechnology 2011, 22 (12), 125706.

(19) Fang, H.; Battaglia, C.; Carraro, C.; Nemsak, S.; Ozdol, B.; Kang, J. S.; Bechtel, H. A.; Desai, S. B.; Kronast, F.; Unal, A. A.; Conti, G.; Conlon, C.; Palsson, G. K.; Martin, M. C.; Minor, A. M.; Fadley, C. S.; Yablonovitch, E.; Maboudian, R.; Javey, A. Proc. Natl. Acad. Sci. U. S. A. 2014, 111, 6198-6202.

(20) Luo, X.; Zhao, Y.; Zhang, J.; Toh, M.; Kloc, C.; Xiong, Q.; Quek, S. Y. Phys. Rev. B: Condens. Matter Mater. Phys. 2013, 88, 195313.

(21) Kresse, G.; Hafner, J. Phys. Rev. B: Condens. Matter Mater. Phys. 1993, 47, R558.

(22) Kresse, G.; Furthmüller, J. Phys. Rev. B: Condens. Matter Mater. Phys. 1996, 54 (16), 11169-11186.

(23) Perdew, J. P.; Burke, K.; Ernzerhof, M. Phys. Rev. Lett. 1996, 77 (18), 3865-3868.

(24) Heyd, J.; Scuseria, G. E.; Ernzerhof, M. J. Chem. Phys. 2006, 124 (21), 9906.

(25) Hedin, L. Phys. Rev. 1965, 139 (3A), A796-A823.

(26) Shi, H.; Pan, H.; Zhang, Y.-W.; Yakobson, B. I. Phys. Rev. B: Condens. Matter Mater. Phys. 2013, 87 (15), 155304.

(27) Cheiwchanchamnangij, T.; Lambrecht, W. R. L. Phys. Rev. B: Condens. Matter Mater. Phys. 2012, 85 (20), 205302.

(28) Sze, S. M.; Ng, K. K. Physics of Semiconductor Devices; WileyVCH: Weinheim, Germany, 2006. 


\section{Strain Induced Indirect to Direct Bandgap Transition}

\section{in Multilayer $\mathrm{WSe}_{2}$}

Sujay B. Desai ${ }^{1,2}$, Gyungseon Seol ${ }^{3}$, Jeong Seuk Kang ${ }^{1,2}$, Hui Fang ${ }^{1,2}$, Corsin Battaglia ${ }^{1,2}$, Rehan Kapadia ${ }^{1,2}$, Joel W. Ager ${ }^{2}$, Jing Guo ${ }^{3}$, and Ali Javey ${ }^{1,2, \dagger}$

${ }^{1}$ Electrical Engineering and Computer Sciences, University of California, Berkeley, CA, 94720.

${ }^{2}$ Materials Sciences Division, Lawrence Berkeley National Laboratory, Berkeley, CA, 94720.

${ }^{3}$ Department of ECE, University of Florida, Gainesville, FL, 32611-6130.

†Corresponding Author:ajavey@eecs.berkeley.edu

\section{Supporting Information}




\section{Sample preparation method:}

$\mathrm{WSe}_{2}$ (2dsemiconductors.com) was mechanically exfoliated using the adhesive tape method onto $260 \mathrm{~nm} \mathrm{SiO} / \mathrm{Si}$ substrate ( $\mathrm{SVM}$ wafers). The chips were mapped to find the flakes of desired thickness using an optical microscope based on the color and contrast of the flakes, which is determined by the thickness of the underlying oxide $(260 \mathrm{~nm})$ and the refractive index of $\mathrm{WSe}_{2}$. The flakes were then transferred by a dry transfer technique ${ }^{1}$ using PMMA (MicroChem) as the transfer medium, onto a clear and flexible PETG substrate of $1.5 \mathrm{~mm}$ thickness. The samples were subsequently annealed at $125^{\circ} \mathrm{C}$ for 5 minutes so that the flake and PMMA handling medium stick to the PETG. The partial thermoforming/softening of the PETG at the annealing temperature helps clamp the flake along with the PMMA enabling the application of large strain. 


\section{Strain Calculation and $\mathrm{MoS}_{2}$ internal reference:}

The bent PETG substrate is approximated as a circular arc for the purpose of strain calculation. A snapshot of the bent PETG is taken at each strain condition and then the parameters ' $\theta$ ' and ' $a$ ' as shown in Fig. 1a are extracted. The strain at the top surface of the PETG is given by $\varepsilon=\frac{\tau}{2 R}$ where $\tau$ is the thickness of the PETG and $\mathrm{R}$ is the radius of the bent PETG. From geometry $R=\frac{a}{\sin \theta}$ which gives $\varepsilon=\frac{\tau \sin \theta}{2 a}$. The computed strain is valid for low angles of ' $\theta$ '. At large ' $\theta$ ' the circular arc approximation fails and the maximum strain is $\sim 20 \%$ higher $^{2}$. For this work, all strain values are computed using the circular arc approximation. The strain computed is also compared with strain calculated from a $\mathrm{MoS}_{2}$ internal reference flake which is discussed in Fig. S1 in more detail.
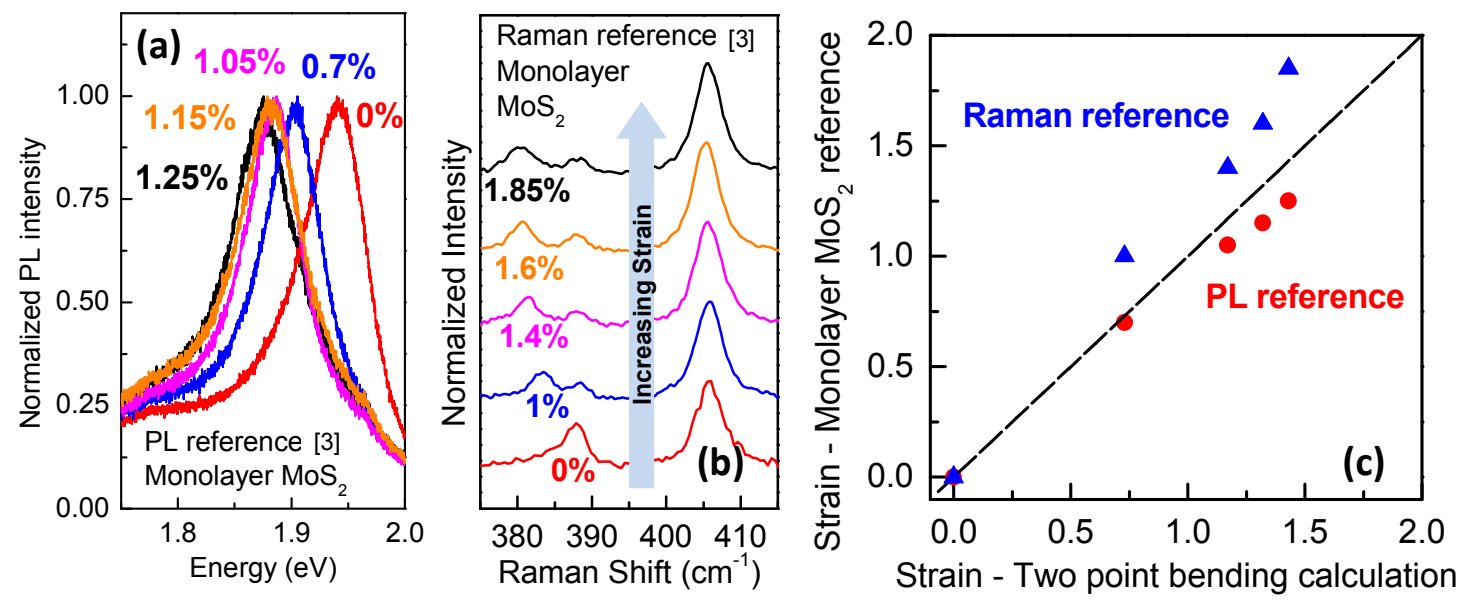

Figure S1: (a) PL spectra for monolayer $\mathrm{MoS}_{2}$ for varying strain. The shift in the PL peak is used to calculate strain from reference ${ }^{3}$. The absolute peak value is different from that in ref. ${ }^{3}$ possibly due to substrate effects (Reference ${ }^{3}$ used polycarbonate whereas we use PETG). (b) Raman spectra for monolayer $\mathrm{MoS}_{2}$ for varying strain. Again the shift in the Raman peak is used to compute strain from reference ${ }^{3}$. (c) The strain from the PL and Raman references in (a) and 
(b) is plotted versus the strain computed for the same samples from our own two-point bending calculation showing an almost 1:1 correlation.

\section{Local Strain effects and reversibility of strain:}

The Raman and PL measurements over multiple spots on a flake show that small local variations in strain exist on a flake even at zero strain. The local strain effects can be due to multiple reasons such as existing defects, ripples, folds or edges in the flake. In addition small strain can also be induced by thicker adjacent flakes or during the processing of the sample. Multiple spots on a flake were measured and representative data discussed in this work represents the modal trends seen at each strain. A PL line scan on a bilayer sample also shows that local strain variations are over a much larger scale than the spot size of the laser.

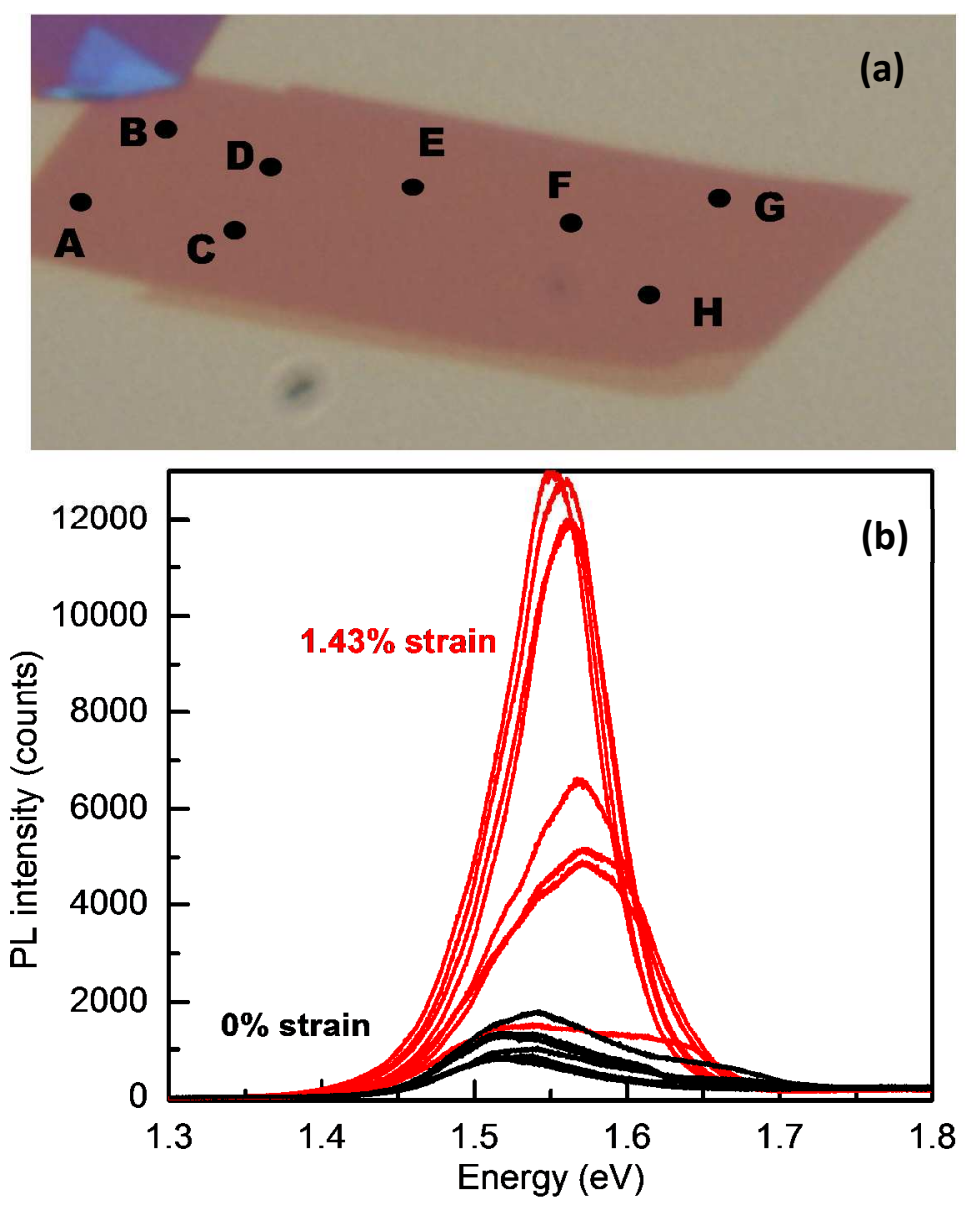


Figure S2: (a) Optical image of bilayer $\mathrm{WSe}_{2}$ flake showing the spots where PL and Raman spectra are measured. (b) PL spectra for different spots at a given strain indicating the variation in PL due to local strain effects.

Repeated straining experiments with PETG samples were also performed to check the reversibility of strain application. This is important so as to verify that the flake does not slip on the substrate and the strain measured is actually the strain imparted to the flake. The reversibility in trends for a bilayer sample were seen up to a strain of $\sim 1.04 \%$. For very large strain, the PETG enters the plastic regime and cannot return to its original unbent shape. 


\section{Density functional theory analysis of $\mathrm{WSe}_{2}$ under uniaxial tensile strain:}

Heyd-Scuseria-Ernzerhof (HSE) exchange correlation potential with spin orbit coupling was used to model the electronic band structure for $\mathrm{WSe}_{2}$. An ultra-soft PAW pseudo-potential with semi-core electrons and a cut-off energy of $400 \mathrm{eV}$ for the plane waves was used. For strained $\mathrm{WSe}_{2}$, structure relaxation was first performed to determine the relaxed atomistic structure in the presence of uniaxial strain, and the band structure in the presence of strain was subsequently calculated. Structural relaxation is achieved by minimizing the energy with a tolerance of $1 \mathrm{meV}$ (for total energy in the range of 44-45eV). Fig. S3 schematically represents the direction of application of strain in the DFT calculations and the reciprocal space of $\mathrm{WSe}_{2}$.

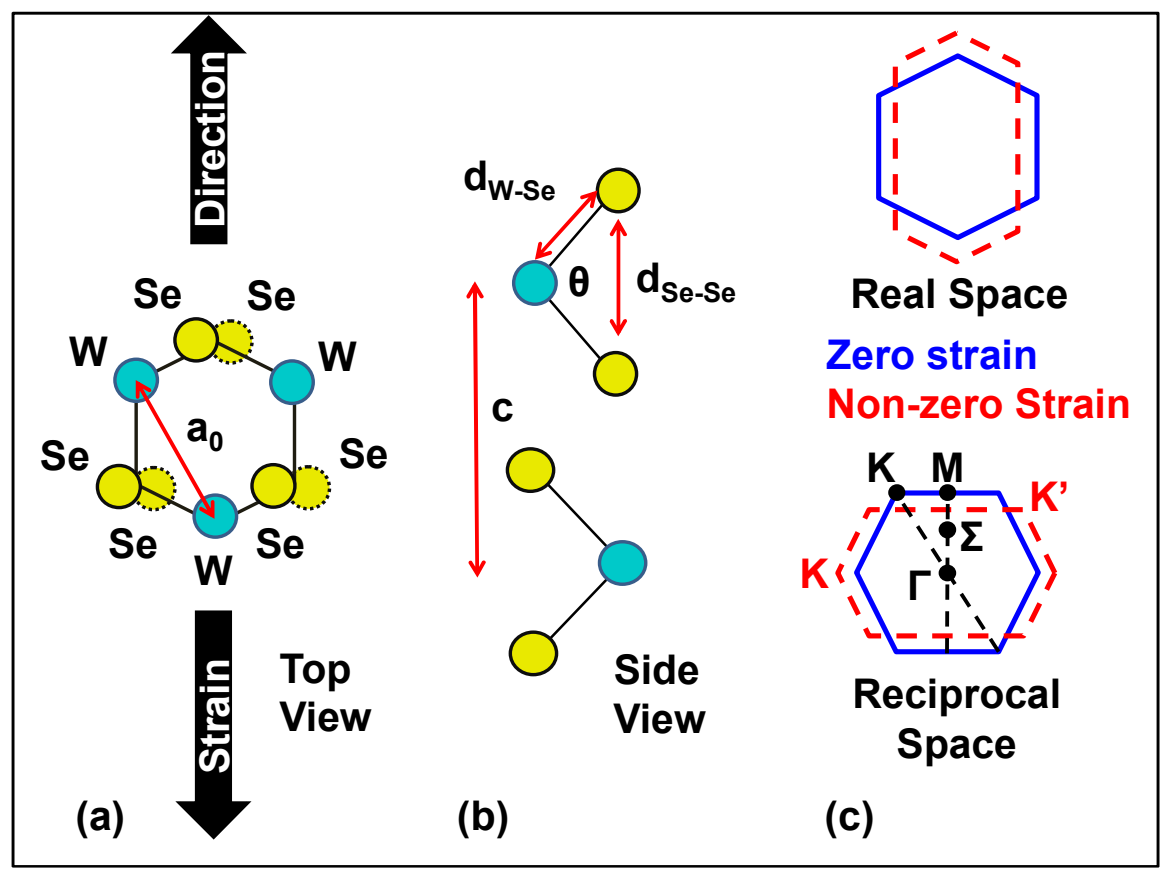

Figure S3: (a) Top view of $\mathrm{WSe}_{2}$ showing direction of strain (b) Side view of $\mathrm{WSe}_{2}$ (c) Real and reciprocal space of $\mathrm{WSe}_{2}$ with and without strain. Reciprocal space shows the $\mathrm{K}, \mathrm{M}, \Sigma$ and $\Gamma$ points.

We see that the crystal lattice and the reciprocal space get stretched when strain is applied. For monolayer $\mathrm{WSe}_{2}$ as strain is applied the intra-layer Se-Se distance decreases; 
whereas for bilayer $\mathrm{WSe}_{2}$ the Se-Se intra-layer and inter-layer distances decreases whereas the $\mathrm{W}$-Se bond length at the outer Se increases. The degeneracy of the $6 \mathrm{~K}$ points is however maintained because the positions of the $\mathrm{K}, \mathrm{M}, \Sigma$, etc. points may have changed, but the E- $k$ relation along two different directions say $\mathrm{K}-\Gamma$ and $\mathrm{K}^{\prime}-\Gamma$ (Fig. S3c) will still be the same because the reciprocal lattice vectors have also changed.
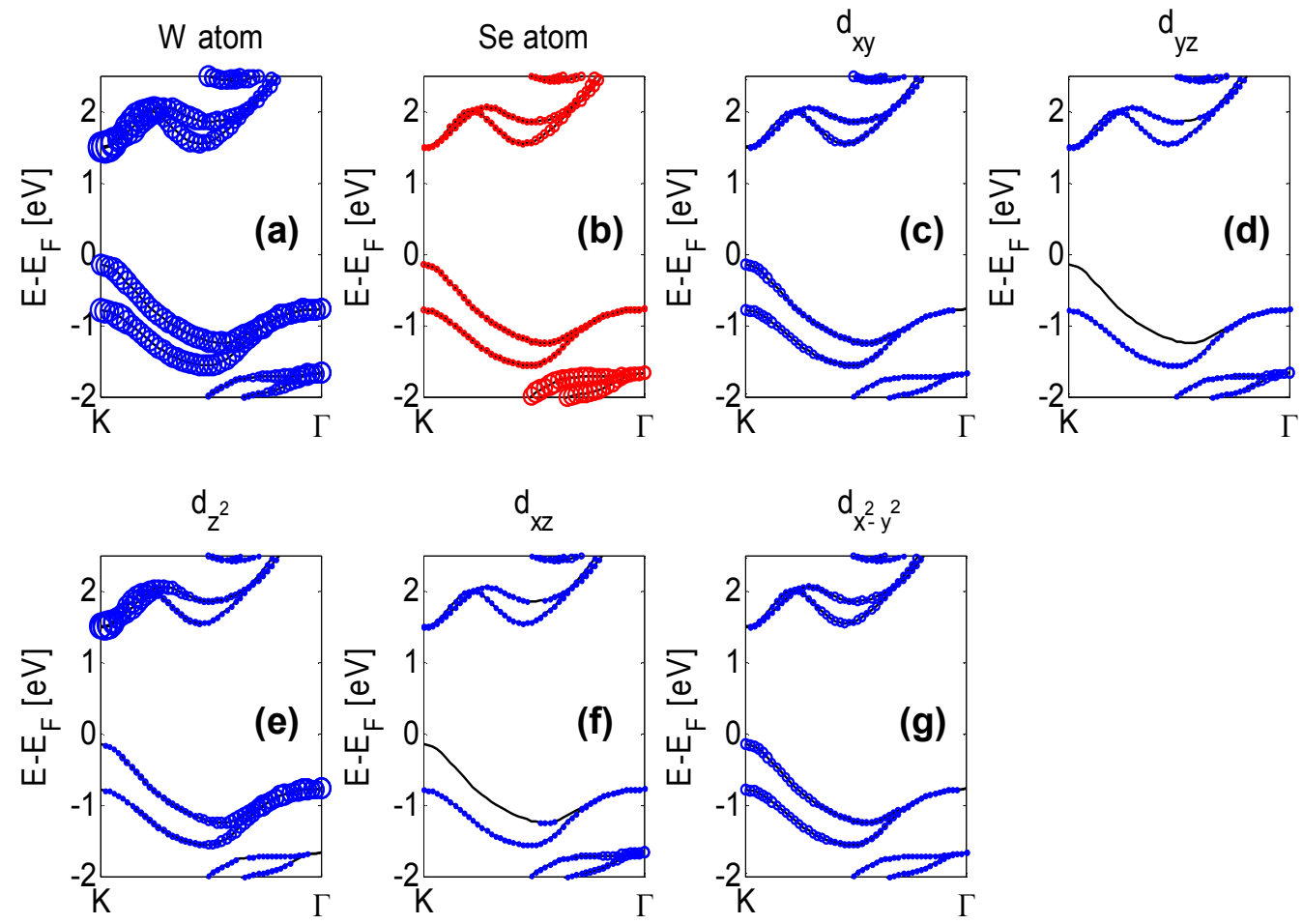

Figure S4: Electronic band structure for unstrained monolayer $\mathrm{WSe}_{2}$ from $\mathrm{HSE}-\mathrm{DFT}$ simulations showing the relative contribution of different orbitals to the energy bands, which is directly proportional to the size of the circles on the curves. (a) W atom orbital and (b) Se atom orbital contributions. (c-g) Different W orbital contributions to the energy bands.

Fig. S4a and Fig. S4b clearly illustrate that the $\mathrm{W}$ atom orbitals dominantly contribute to the energy bands near the conduction band (CB) minima and the valence band (VB) maxima. From Fig. S4c to Fig. S4g we also understand that the contribution of the $\mathrm{W} \mathrm{d}_{\mathrm{Z}^{2}}$ orbital to the $\mathrm{CB}$ 
minima at the $\mathrm{K}$ point is maximum. The above observations are found to be true for bilayer $\mathrm{WSe}_{2}$ as well from the DFT calculations. Thus change of the interlayer W-W distance in strained bilayer $\mathrm{WSe}_{2}$ and hence the orbital overlap for the $\mathrm{d}_{\mathrm{Z}^{2}}$ orbitals affects the CB minima at the K point drastically and leads to an indirect to direct bandgap transition as explained in Fig. $2 b$ in the main text. 


\section{Effect of uniaxial tensile strain on monolayer WSe $\mathrm{W}_{2}$}

Monolayer $\mathrm{WSe}_{2}$ PL spectra at different strain in Fig. 4a clearly show an increase in PL intensity with increasing strain. Also peak shifts and FWHM becomes narrower. For no strain case the PL peak is at $1.7 \mathrm{eV}$ (shifted from $1.65 \mathrm{eV}$ when on $\mathrm{SiO}_{2}$ ) and also shows a significant red tail. This can be possibly due to substrate and excitonic effects in $\mathrm{WSe}_{2}$. Raman spectra at different strain for monolayer $\mathrm{WSe}_{2}$ in Fig. $4 \mathrm{~b}$ show peak splitting of the $\mathrm{E}_{2 \mathrm{~g}}^{1}$ mode indicating that strain is applied on the flake.
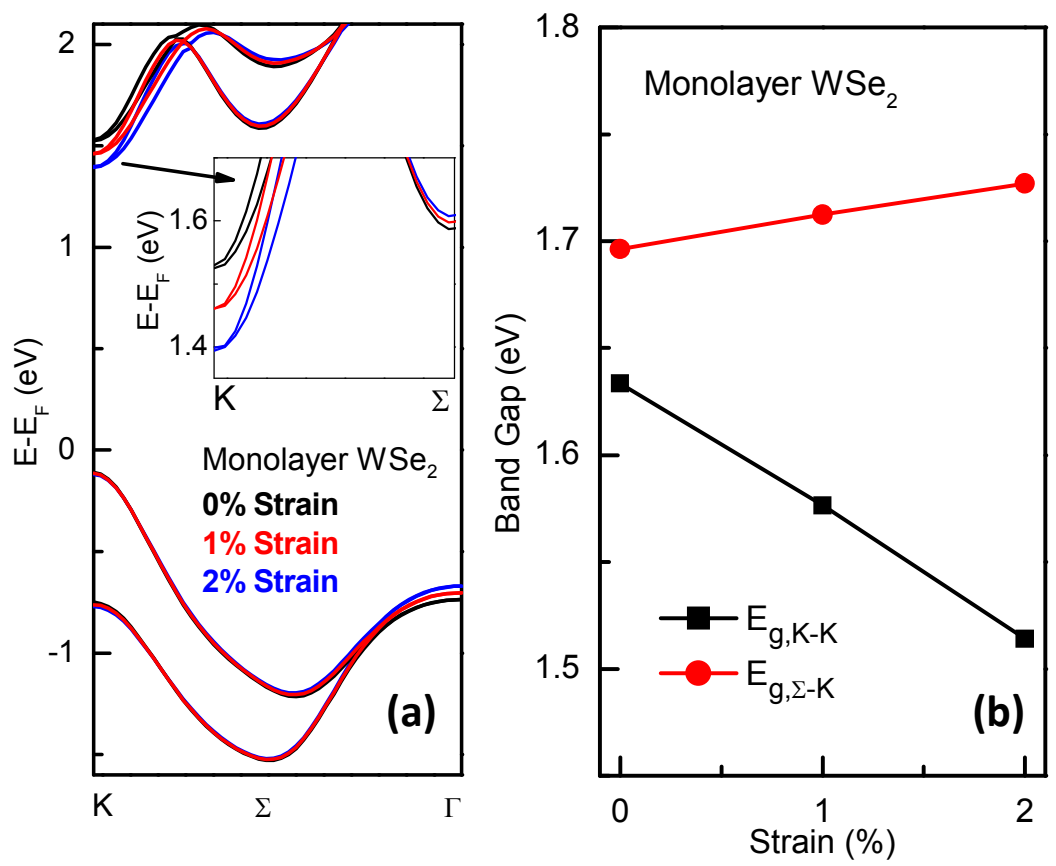

Figure S5: (a) Electronic band structure for monolayer $\mathrm{WSe}_{2}$ from $\mathrm{HSE}-\mathrm{DFT}$ simulations showing the bandstructure $\mathrm{w}$ and w/o strain. $\mathrm{CB}$ minima at $\mathrm{K}$ point moves down drastically indicating that monolayer $\mathrm{WSe}_{2}$ remains direct bandgap even under tensile strain and the difference between the indirect and the direct valleys increases further. (b) Indirect and direct bandgap values obtained from HSE-DFT simulations for monolayer $\mathrm{WSe}_{2}$. 
Raman and PL spectra at different strain for bulk WSe ${ }_{2}$ :
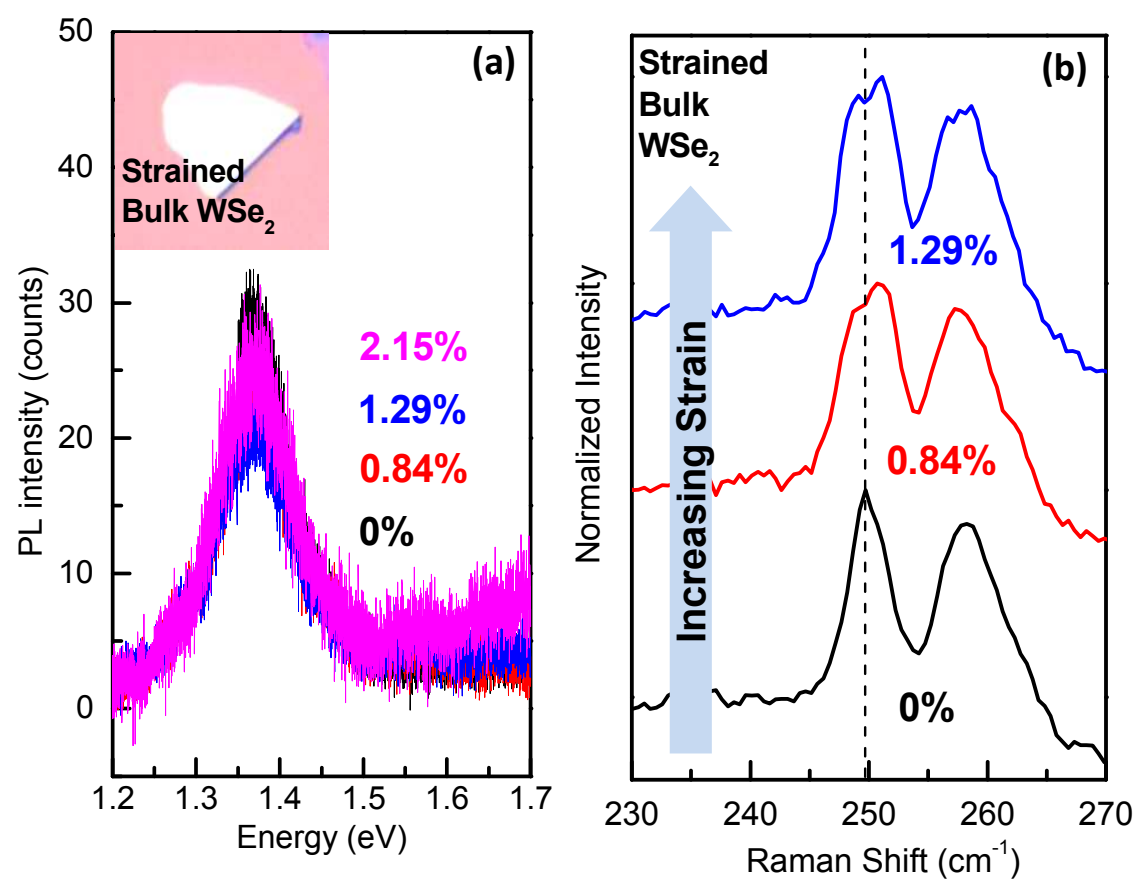

Figure S6: (a) PL and (b) Raman spectra at different strain for bulk $\mathrm{WSe}_{2}$. Inset shows corresponding flake. Peak splitting seen in all spectra at non-zero strain. PL spectra at different strain for bulk $\mathrm{WSe}_{2}$ show no change in PL consistent with the fact that for bulk $\mathrm{WSe}_{2}$ the direct and indirect bandgap difference is very large $(\sim 200 \mathrm{meV}){ }^{4}$, thus requiring huge strain for a crossover. 


\section{$\mathbf{G}_{0} \mathbf{W}_{0}$ calculation approach:}

In the following GW quasi-particle (QP) calculation, non-self-consistent $\mathrm{G}_{0} \mathrm{~W}_{0}$ was used to reduce the computational cost ${ }^{5}$. Same atomistic relaxed structure was used as in the main text. HSE exchange-correlation functional was used to obtain wave functions for the GW calculation. Although this step is not essential, this method has been suggested to improve agreement with experiments ${ }^{6}$. The Brillouin zone sampled with a $6 \times 6 \times 1$ k-point mesh. The band-structure was Wannier interpolated using the WANNIER90 program ${ }^{7}$. From the figure $\mathrm{S} 7 \mathrm{a}, \sim 0.65 \mathrm{eV}$ shift of $\mathrm{CBM}$ as regard to the valence band can be observed. In figure S7b, splitting the valence bands due to spin orbital coupling is shown.
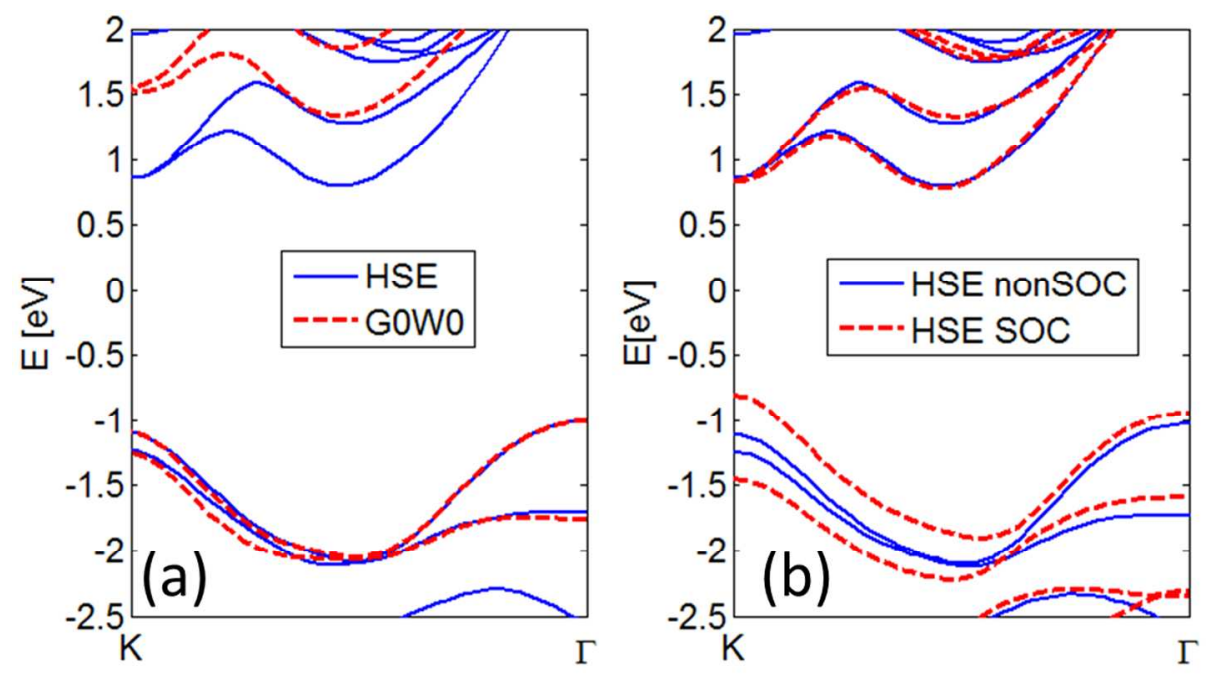

Figure S7: (a) Comparison of Wannier interpolated HSE-DFT and $\mathrm{G}_{0} \mathrm{~W}_{0}$ calculation. SOC is not considered and no strain is applied. (b) Comparison of spin orbital coupling (SOC) effect in HSE-DFT calculation. Band splitting in the K point of the valence band can be observed. 


\section{REFERENCES}

1. Fang, H.; Battaglia, C.; Carraro, C.; Nemsak, S.; Ozdol, B.; Kang, J. S.; Bechtel, H. A.;

Desai, S. B.; Kronast, F.; Unal, A. A.; Conti, G.; Conlon, C.; Palsson, G. K.; Martin, M. C.;

Minor, A. M.; Fadley, C. S.; Yablonovitch, E.; Maboudian, R.; Javey, A. Proc. Natl. Acad. Sci. 2014.

2. Matthewson, M. J.; Kurkjian, C. R.; Gulati, S. T. Jour. Amer. Ceramic Soc. 1986, 69, (11), 815-821.

3. Conley, H. J.; Wang, B.; Ziegler, J. I.; Haglund, R. F.; Pantelides, S. T.; Bolotin, K. I. Nano Lett. 2013, 13, (8), 3626-3630.

4. Sahin, H.; Tongay, S.; Horzum, S.; Fan, W.; Zhou, J.; Li, J.; Wu, J.; Peeters, F. M. Phys. Rev. B 2013, 87, (16), 165409.

5. Hedin, L. Phys. Rev. 1965, 139, (3A), A796-A823.

6. Ramasubramaniam, A. Phys. Rev. B 2012, 86, (11), 115409.

7. Mostofi, A. A.; Yates, J. R.; Lee, Y.-S.; Souza, I.; Vanderbilt, D.; Marzari, N. Computer Phys. Comm. 2008, 178, (9), 685-699. 